\title{
因HAD
}

DOI: http://doi.org/10.22585/hospdomic.v5i1.111

\section{Unidad de Hospitalización a Domicilio del paciente quirúrgico durante la pandemia de la COVID-19}

\section{Home Hospitalization Unit of Surgical Patients During the COVID-19 Pandemic}

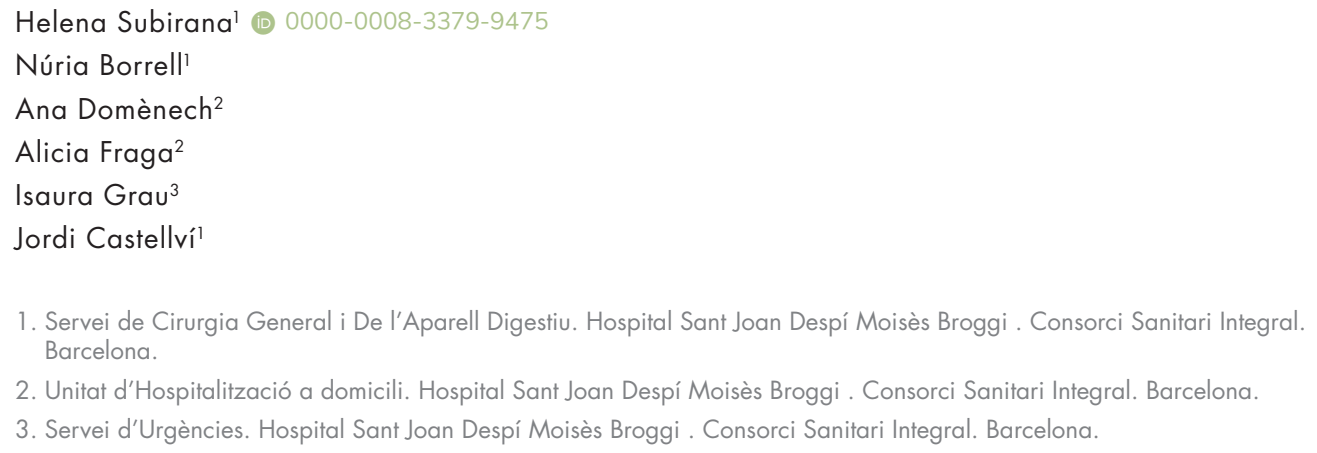

Correspondencia/Correspondence

Helena Subirana Magdaleno

Helena.subirana@gmail.com

Recibido/Received

15.07.2020

Aceptado/Accepted

16.11.2020

Financiación/Funding

Sin financiación.

\author{
Conflicto de Intereses/Competing interest \\ Las autoras y el autor declaran no tener ningún conflicto de \\ intereses. \\ Contribuciones de autoría/Author contributions \\ Las autoras y el autor han contribuido por igual en la \\ realiczación de este trabajo.
}

CÓMO CITAR ESTE TRABAJO | HOW TO CITE THIS PAPER

Subirana H, Borrell N, Domènech A, Fraga A, Grau I, Castellví J. Unidad de Hospitalización a Domicilio del paciente quirúrgico durante la pandemia de la COVID-19. Hosp Domic. 2021;5(1):9-16. 


\section{RESUMEN}

Introducción: la pandemia de la COVID-19 ha puesto a prueba sistemas sanitarios a nivel mundial. Desde el servicio de Cirugía General y ante la disminución de camas de hospitalización surgió la necesidad de planificar y organizar una estrategia para la hospitalización y cuidado de pacientes quirúrgicos.

La hospitalización a domicilio (HAD) es una modalidad asistencial eficaz en patología médica y quirúrgica donde, por un tiempo limitado, personal sanitario realiza tratamiento y seguimiento de pacientes en su domicilio.

Objetivo: describir nuestra experiencia de HAD en el paciente quirúrgico durante la pandemia de la COVID-19.

Método: estudio observacional, descriptivo, prospectivo, unicéntrico. 44 pacientes en HAD del 11 de Marzo al 10 de Mayo de 2020. Se incluyeron pacientes sometidos a cirugía urgente, programada y pacientes quirúrgicos con tratamiento conservador o intervencionismo. Se evaluaron variables epidemiológicas, motivo de ingreso, tratamiento, estancia media, tasa de reingresos, complicaciones y mortalidad.

Resultados: edad media 64 años \pm 16,7 (23-89). $52 \%$ hombres y $49 \%$ mujeres. 23 pacientes con cirugía urgente o programada, 12 tratamiento conservador, 2 drenaje torácico, 5 drenaje percutáneo y 2 terapia de presión negativa para cura de herida quirúrgica. Estancia media 8,6 días $\pm 3,6$ (1-19). Registradas 5 complicaciones: suboclusión, rectorragia, enfisema subcutáneo, absceso pulmonar e infección por COVID-19. 2 pacientes reingresaron.

Conclusiones: La HAD es un modelo seguro y eficaz para disminuir la estancia hospitalaria en pacientes quirúrgicos seleccionados. La combinación de ingreso de corta estancia e ingreso en régimen de HAD podría ser una buena opción en el postoperatorio y recuperación de pacientes quirúrgicos.

Palabras clave: Servicios de Atención de Salud a Domicilio; Hospitalización a domicilio; Virus SRAS-COV2; COVID-19; Cuidados Posoperatorios; Paciente quirúrgico.

\section{ABSTRACT}

Introduction: current COVID-19 pandemic is testing healthcare systems at a global level. From General Surgery Department we decided to provide a practical strategy for surgical patients' hospitalization that allowed to treat them as if they were admitted in hospital.

Home Hospitalization is an effective healthcare modality in medical and surgical pathology in which, for a limited time, health practitioners provide active treatment at patient's home.

The aim of this study is to describe our experience of home care hospitalization in surgical patients during the covid-19 pandemic.

Method: Observational, descriptive, prospective, single-center study. 44 patients admitted to surgical home hospitalization unit from March 11 to May 10 2020. Patients with emergency or elective surgery and surgical patients who required conservative medical treatment or interventionism were included. Epidemiological variables, diagnosis, treatment, mean stay, readmissions rate, complications and mortality were evaluated.

Results: Mean age 64 years $\pm 16,7$ (23-89). 52\% men and 49\% women. 23 patients underwent emergency or elective surgery, 12 patients conservative medical treatment, 2 thoracic drainage, 5 percutaneous drainage due to intra-abdominal abscesses and 2 patients with negative pressure therapy for surgical wound healing. Mean hospital stay 8,6 days $\pm 3,6$ (1-19).

Five complications were registered: subocclusion, colo-rectal bleeding, subcutaneous emphysema, lung abscess and a COVID-19 infection. Readmissions rate: $4,5 \%$.

Conclusions: Home hospitalization is an effective model to decrease or avoid hospital stay in selected patients. It also appears to be as safe as inpatient hospitalization. Combination of short-stay admission and home admission could be a good therapeutic option in surgical patients.

Keywords: Home Care Services; Hospital at home care; SARS- COV2 Virus; COVID-19; Postoperative Care; Surgical patient. 


\section{INTRODUCCIÓN}

El 11 de Marzo de 2020 la Organización Mundial de la Salud (OMS) declaró pandemia global la infección por COVID-19, clasificándola como una emergencia internacional. La gran capacidad de transmisión del virus puso en entredicho la efectividad y la sostenibilidad de nuestros sistemas de salud $^{(1)}$.

La gran cantidad de pacientes recibidos en las urgencias de nuestros centros, el incremento de volumen de los ingresos hospitalarios y el alto riesgo de exposición al virus hicieron que las principales organizaciones recomendaran la cancelación de las cirugías electivas en los hospitales ${ }^{(2)}$.

A pesar de ello, las urgencias quirúrgicas han seguido siendo atendidas y tratadas en nuestros servicios de urgencias y algunas neoplasias no demorables han sido intervenidas.

La hospitalización a domicilio es un modelo instaurado desde hace algunos años en equipos no quirúrgicos. Es eficaz y eficiente en la atención al paciente (los resultados son comparables a la hospitalización convencional en pacientes seleccionados), segura (minimiza infecciones nosocomiales, disminuye la realización de pruebas complementarias innecesarias) y con un alto índice de satisfacción ${ }^{(3)}$.

Nuestro objetivo fue describir el nuevo modelo de hospitalización a domicilio (HAD) quirúrgico recientemente instaurado en nuestro centro durante el periodo de pandemia de la COVID-19 y analizar los resultados preliminares.

\section{MÉTODOS}

Se diseñó y realizó un estudio descriptivo, prospectivo, observacional, unicéntrico.

Entre el 11 de Marzo y el 10 de Mayo de 2020 fueron ingresados en régimen de hospitalización a domicilio 44 pacientes del Servicio de Cirugía General y Digestiva.

Los criterios de inclusión fueron pacientes mayores de 18 años con un diagnóstico de patología de régimen quirúrgico en tratamiento conservador, postoperatorio de cirugía urgente o programada y todos ellos con estabilidad clínica, presencia de cuidador principal en el domicilio las 24 horas, y con unas condiciones de hábitat higiénicas y socio-familiares adecuadas así como disponibilidad de comunicación telefónica.

Se excluyeron los pacientes con adicción a drogas por vía parenteral o alcoholismo severo, sociopatía o indigencia, domicilio fuera del área de influencia y la no aceptación por parte del paciente o cuidador.

El cirujano responsable del paciente, desde consultas externas, urgencias u hospitalización realiza interconsulta al servicio de HAD quirúrgico quien valora al paciente y evalúa si cumple los criterios de inclusión. Una vez aceptado el paciente se define y consensua el tratamiento a seguir entre los dos especialistas.

Todos los pacientes firmaron el consentimiento informado específico de ingreso en HAD.

El equipo de HAD quirúrgico estaba formado por una enfermera clínica especializada en paciente quirúrgico y un cirujano general y del aparato digestivo, que atienden un máximo de 10 pacientes.

La atención o visita domiciliaria fue diaria de 8 a 20h de lunes a viernes. Se les ofrece contacto telefónico las 24 horas del día. Durante el fin de semana la visitas se restringen dependiendo de las necesidades. 
Las variables de estudio fueron edad y sexo, motivo de ingreso, tratamiento realizado, estancia media en HAD, tasa de reingresos, complicaciones y mortalidad.

Se realizó un análisis descriptivo de las variables del estudio. Las variables continuas se describieron como media y desviación estándar (DE) o como mediana y rango; y las variables categóricas como frecuencias absolutas y porcentajes. Para la recogida de datos y el análisis estadístico se utilizó una base de datos Microsoft@ Access 2007 y el programa SPSS v16.

\section{RESULTADOS}

De 204 pacientes ingresados en el Servicio de Cirugía general, 44 (22\%) fueron atendidos en régimen de HAD. De ellos, 31(70\%) procedentes de planta de hospitalización, 11 (25\%) ingresados desde Urgencias y 2 pacientes (5\%) de Consultas externas.

La edad media es de 64 años $\pm 16,7$ (23-89). 52\% varones y 49\% mujeres (tabla 1 ).

Tabla 1. Resultados epidemiológicos (N: 44)

\begin{tabular}{|l|l|}
\hline Edad/años \pm DS (rango) & $64 \pm 16,7(23-89)$ \\
\hline Sexo & $23(49 \%)$ Mujeres \\
\cline { 2 - 2 } & $21(52 \%)$ Hombres \\
\hline
\end{tabular}

Fueron operados 23 pacientes, 7 pacientes de cirugía urgente y 16 de cirugía programada. 12 pacientes recibieron tratamiento médico conservador, 2 pacientes portadores de drenaje torácico, 5 tras colocación de drenaje percutáneo por abscesos intraabdominales y 2 pacientes con terapia de presión negativa (tabla 2).

Tabla 2. Motivo de ingreso (N: 44)

\begin{tabular}{|l|l|}
\hline Cirugía programada o urgente diferida (16) & $\begin{array}{l}\text { Neoplasia de recto 7 } \\
\text { Neoplasia de colon derecho } 2 \\
\text { Neoplasia de sigma 2 } \\
\text { Neoplasia de colon transverso 1 } \\
\text { Diverticulitis con fistula colovesical 1 } \\
\text { Neoplasia de mama 3 }\end{array}$ \\
\hline Cirugía urgente (7) & $\begin{array}{l}\text { Apendicetomía 2 } \\
\text { Plastrón apendicular 1 } \\
\text { Colecistectomía 1 } \\
\text { Absceso mamario 1 } \\
\text { Oclusión intestinal 1 } \\
\text { Absceso de pared abdominal 1 }\end{array}$ \\
\hline
\end{tabular}




\begin{tabular}{|l|l|}
\hline Tratamiento médico conservador (12) & $\begin{array}{l}\text { Diverticulitis aguda 7 } \\
\text { Plastrón apendicular } 1 \\
\text { Absceso cutáneo 1 } \\
\text { Colangitis con absceso hepático 1 } \\
\text { Colecistitis aguda 1 } \\
\text { Suboclusión intestinal 1 }\end{array}$ \\
\hline Drenaje torácico (2) & $\begin{array}{l}\text { Neumonía necrotizante + neumotórax 1 } \\
\text { Neumonía con fuga persistente post - COVID1 }\end{array}$ \\
\hline Drenaje percutáneo (5) & $\begin{array}{l}\text { Pancreatitis aguda con abscesos 2 } \\
\text { Colecistitis aguda - colecistostomia 1 } \\
\text { Plastrón apendicular con absceso 1 } \\
\text { Absceso hepático 1 }\end{array}$ \\
\hline Terapia presión negativa (2) & $\begin{array}{l}\text { Dehiscencia herida perineal } 1 \\
\text { Absceso pared abdominal 1 }\end{array}$ \\
\hline
\end{tabular}

La estancia media global en hospitalización a domicilio fue de 8,6 días \pm 3,6 (1-19).

Los pacientes intervenidos, tanto de cirugía urgente como programada tuvieron una estancia hospitalaria convencional media de 4,3 días (0-21).

Recibieron tratamiento antibiótico durante la hospitalización a domicilio 24 pacientes (54.5\%), el $58 \%$ de ellos por vía endovenosa y el $42 \%$ por vía oral.

Se registraron complicaciones en 5 pacientes durante el ingreso en HAD. Un caso de suboclusión intestinal a los 10 días postoperatorios de hemicolectomía derecha por neoplasia de colon que se resolvió con tratamiento conservador, rectorragia en el $6^{\circ}$ DPO de hemicolectomía derecha visitada en urgencias, enfisema subcutáneo en paciente portador de drenaje torácico precisando visita y control radiológico en urgencias, infección de herida quirúrgica y COVID-19 y absceso pulmonar (tabla 3).

Tabla 3. Resultados (N: 44)

\begin{tabular}{|l|l|}
\hline Estancia media / días \pm DS (rango) & $8,6 \pm 3,6(1-19)$ \\
\hline Complicaciones & $\mathbf{5 ( 1 1 \% )}$ \\
\hline Grado I Clavien Dindo & $\begin{array}{l}\text { - Enfisema Subcutaneo } \\
\text { - Suboclusión intestinal }\end{array}$ \\
\hline
\end{tabular}




\begin{tabular}{|l|l|}
\hline Grado II Clavien Dindo & - Rectorragia \\
\hline Grado III Clavien Dindo & $\begin{array}{l}\text { - Infección COVID19 neumonía } \\
\text { bilateral (ingreso) } \\
\text { - Absceso pulmonar (ingreso) }\end{array}$ \\
\hline
\end{tabular}

Reingresaron 2 pacientes (4,5\%): paciente con antecedente de colecistectomía y exploración de la vía biliar, portador de drenaje biliar que presentó infección de herida quirúrgica e infección por COVID-19 con neumonía bilateral grave requiriendo ingreso durante 3 semanas y un paciente con absceso hepático que fue drenado con pigtail y presentó absceso pulmonar por contigüidad que requirió drenaje ecoguiado con buena evolución posterior.

\section{DISCUSIÓN}

La pandemia de la COVID-19 ha saturado el sistema sanitario y con ello ha disminuido la capacidad de poder asumir y ofrecer a los pacientes los cuidados necesarios que precisen en función de su patología. La necesidad de disponer de camas de hospitalización tanto de pacientes críticos como en planta ha obligado a los profesionales sanitarios a buscar alternativas a la hospitalización convencional(4).

La hospitalización a domicilio se ha descrito como un modelo útil para acortar la estancia hospitalaria y se basa en dos principales pilares: evitar los ingresos hospitalarios y permitir una alta precoz ${ }^{(5)}$, liberando así camas hospitalarias.

En los dos modelos, el estrecho contacto con el centro hospitalario y un equipo médico especializado, es fundamental y puede beneficiar a los pacientes en un momento como el actual ${ }^{(6,7)}$.

De 204 pacientes ingresados por patología quirúrgica en el servicio de Cirugía General el 22\% se pudieron acoger al sistema de hospitalización a domicilio disminuyendo la estancia en hospitalización convencional, hecho que ha permitido tener camas disponibles para otros pacientes.

Se han conseguido liberar 6,2 camas de hospitalización convencional por día durante el período estudiado.

También se han conseguido disminuir el número de ingresos hospitalarios ya que un tercio de los pacientes que fueron ingresados en la Unidad de HAD lo hicieron directamente desde urgencias, sin pasar por la planta de hospitalización.

Sin embargo, la gran variabilidad en los diagnósticos y tratamientos de los pacientes durante este periodo no nos permite demostrar una disminución de la estancia hospitalaria comparándola con la hospitalización convencional, aunque si tenemos la percepción que se han dado altas más precoces de lo habitual, sobre todo en los pacientes sometidos a cirugías programadas.

La estancia media de hospitalización convencional de los pacientes que ingresaron en HAD fue de 4,3 días. En el mismo período del año anterior la estancia media de hospitalización convencional fue de 5,18 días. Así pues, a pesar de la complejidad de los pacientes ingresados durante la pandemia se ha conseguido disminuir la estancia media de hospitalización convencional.

Es importante remarcar que serían necesarios estudios comparativos en pacientes con el mismo diagnóstico y procedimientos con ingreso convencional. 
La HAD tiene la ventaja de disminuir el riesgo de infecciones nosocomiales, una recuperación más temprana y una reducción de los costes hospitalarios ${ }^{(8)}$.

En esta situación de pandemia, la hospitalización a domicilio ha permitido reducir los contactos intrahospitalarios y en consecuencia disminuir el riesgo de infecciones nosocomiales habitual y por SARS-CoV-2.

En nuestra experiencia, solo un paciente presentó infección por COVID-19 probablemente adquirida en el centro hospitalario.

Las posibilidades de atención de los pacientes quirúrgicos en las unidades de hospitalización a domicilio son diversas e incluyen postoperatorios de cirugías con alta precoz, las complicaciones quirúrgicas y tratamientos médicos ${ }^{(9)}$.

Durante los ingresos en HAD se realizaron cuidados de rango hospitalario como curas complejas, control y lavados de drenajes intraabdominales, pruebas específicas de laboratorio, antibioterapia endovenosa, ofreciendo el mismo tratamiento que se les habría realizado en el centro hospitalario y con una mejor recuperación en su entorno, ya que les proporciona una sensación de mayor seguridad.

Un $28 \%$ de los pacientes fueron ingresados a HAD con un drenaje intraabdominal post- intervención o por abscesos intraabdominales. Aunque los pacientes no son homogéneos en cuanto al diagnóstico y tratamiento la media de retirada de los drenajes fue a los 9 días posteriores a su colocación. Además de proporcionarles el tratamiento adecuado durante esos días, estos pacientes evitaron el control precoz de la primera visita postoperatoria en consultas externas.

Las complicaciones evidenciadas durante los ingresos son iguales o menores a grado III en la clasificación de Clavien Dindo(10). Una vez analizadas, ninguna de ellas ha sido consecuencia por la atención a domicilio y también habrían aparecido en régimen de hospitalización convencional.

El hecho de ser un equipo formado por una enfermera quirúrgica y un cirujano general creemos que han sido claves para lograr un manejo integral del paciente y en el diagnóstico temprano de las posibles complicaciones.

La hospitalización a domicilio a pesar de ser una modalidad de atención al paciente eficaz, eficiente, segura y con una alta tasa de satisfacción no está ampliamente aceptada y su implementación es irregular y variable debido a la gran variedad de modelos y recursos y una falta de estudios exhaustivos que confirmen sus resultados ${ }^{(11)}$.

A pesar de ello, en el metaanálisis de Caplan et al., refieren que la hospitalización a domicilio se asocia a una reducción en la mortalidad así como en las tasas de reingreso y los $\operatorname{costes}^{(3)}$.

En nuestro caso reingresaron dos pacientes y no hubo ningún caso de mortalidad.

Son necesarios estudios comparativos que demuestren todos estos resultados. Tampoco hay datos ni evidencia del manejo del paciente quirúrgico en régimen de HAD durante esta emergencia sanitaria y no se han comunicado experiencias sobre la eficacia de este recurso en pacientes postoperados ni en pacientes con complicaciones postquirúrgicas ${ }^{(12)}$.

Mantener la calidad asistencial en régimen de Hospitalización a domicilio con unos menores costes es un reto que está en nuestras manos y que una vez demostrado tendrá una gran proyección de futuro.

En los pacientes quirúrgicos podría considerarse una buena opción la combinación de la cirugía de corta estancia y la hospitalización a domicilio.

La crisis sanitaria que ha ocasionado el SARS-CoV-2 nos condiciona a emprender una innovación en todos los campos, entre ellos la creación de una HAD quirúrgica, cuya finalidad será ser más eficaces, a la vez que reducimos los costes, siempre sin disminuir la calidad asistencial. Esta innovación implica la oportunidad de un cambio considerable en el modelo asistencial que los propios profesionales debemos liderar. 


\section{BIBLIOGRAFÍA}

1. Stahel FP. How to risk-stratify elective surgery during the COVID-19 pandemic? Patient Saf Surg 2020;14:8. DOI: 10.1186/s13037-020-00235-9; PMID: 32288785

2. Flemming S, Hankir M, Ernestus RI, Seyfried F, Germer CT, Meybohm P, et al. Surgery in times of COVID-10 recommendations for hospital and patient management. Langenbecks Arch Surg. 2020;405(3):359-64. DOI: 10.1007/s00423-020-01888; PMID: 32385568

3. Caplan GA, Sulaiman NS, Mangin DA, Aimonino RN, Wilson AD, Barclay L. A metaanalysis of "hospital in the home". Med J Aust. 2012;197(9):512-9. DOI: 10.5694/mja12.10480; PMID: 23121588

4. Álvarez Gallego M, Gortázar de las Casas S, Pascual Migueláñez S, Rubio-Pérez I, Barragán Serrano C, Álvarez Peña E, et al. Impacto de la pandemia por SARS-CoV-2 sobre la actividad y profesionales de un Servicio de Cirugía General y del Aparato Digestivo en un hospital terciario. Cir Esp. 2020;98(6):320-7. DOI: 10.1016/j.ciresp.2020.04.001; PMID: 32336467

5. Spuch JA, Mirón M, Florit L, Escuder J, Castellote M, Zornoza A. Tratamiento domiciliario de pacientes con fístula pancreática. Cir Esp 2008;83(3):129-33. DOI: 10.1016/S0009-739X(08)70527$X$

6. Salazar A, Estrada C, Porta, Lolo M, Tomas S, Alvarez M. Home hospitalization unit: an alternative to standard inpatient hospitalization from the emergency department. Eur J Emerg Med. 2009;16(3):121-3. DOI: 10.1097/MEJ.0b013e32831cbae2; PMID: 19262397

7. Varney J, Weiland TJ, Jelinek G. Efficacy of hospital in the home services providing care for patients admitted from emergency departments: an integrative review. Int J Evid Based Healthc. 2014;12(2):128-41. DOI: 10.1097/XEB.0000000000000011; PMID: 24945961

8. Shepperd S, Doll H, Broad J, Gladman J, lliffe S, Langhorne P, et al. Early discharge hospital at home. Cochrane Database Syst Rev. 2009;(1):CD000356. DOI: 10.1002/14651858.CD000356. pub3; PMID: 19160179

9. Millet M, Carrera JA, Garde C, Goenaga M, Arzelus E, Cuende A. Hospitalización a domicilio y uso inadecuado de la hospitalización en cirugía general. Cir Esp. 2006;79(3):194. DOI: 10.1016/ S0009-739X(06)70852-1

10. Dindo D, Demartines N, Clavien PA. Classification of Surgical Complications. A new proposal with evaluation in a cohort of 6336 patients and results of a survey. Ann Surg 2004;240(2):20513. DOI: 10.1097/01.sla.0000133083.54934.ae; PMID: 15273542

11. Jacobs JM, Hammerman-Rozenberg R, Stessman J. Home Hospitalization: 15 years of experience. Ann Intern Med. 2006;144(6):456. DOI: 10.7326/0003-4819-144-6-200603210-00023; PMID: 16549867

12. Mias MC, Pérez-Ruiz L, Rodriguez S, Massoni A, Solá R, Ros S. Impacto de la hospitalización a domicilio en la cirugía tiroidea: estudio comparativo con la hospitalización convencional. Rev Calidad Asistencial 2002;17(8):619-23. 